\title{
Promises, promises, and precision medicine
}

\author{
Michael J. Joyner ${ }^{1}$ and Nigel Paneth ${ }^{2}$ \\ 'Department of Anesthesiology and Perioperative Medicine, Mayo Clinic, Rochester, Minnesota, USA. '2Departments of Epidemiology and Biostatistics and Pediatrics and Human Development, \\ Michigan State University, College of Human Medicine, East Lansing, Michigan, USA.
}

\section{Introduction}

In 1999 Francis Collins published a foundational document of precision medicine entitled "Medical and Societal Consequences of the Human Genome Project,"(1) which made predictions about the ways the human genome would be used to predict, prevent, and treat disease in 2010. In 2000, he suggested that "Over the longer term, perhaps in another 15 or 20 years, you will see a complete transformation in therapeutic medicine" (2).

The vision described in the article became the aspirational template for the precision medicine movement (Figure 1). We have passed the 2010 deadline and are rapidly approaching 2020, yet the "complete transformation in therapeutic medicine" has not occurred. Using the framework of the predictions made nearly 20 years ago, we argue that the foundational assumptions of precision medicine are unsound.

The terms precision medicine and personalized medicine have been used interchangeably to refer to the view that incorporating information encoded in the human genome as the dominant factor in the prediction, diagnosis, and treatment of human disease will lead to marked improvements in human health. Recently, some precision medicine advocates have recommended expanding the scope of precision medicine to incorporate inputs beyond the genome (3), but because precision medicine has been nearly synonymous with genomics, the emphasis on the genome is our focus (4).

\section{A genetic revolution in medicine?}

Disease with a genetic component. Precision medicine asserts a tight linkage between individual variability in DNA sequence and disease causation. For rare diseases, DNA sequencing has improved the clinical evaluation of many patients. Yet interventions making use of this new information have been limited, and a problematic side effect, especially for family members, is that estimates of the penetrance of pathogenic DNA variants decline as more unaffected individuals are screened. Reclassification of variants initially thought to be pathogenic has proven to be a common problem (5).

A few gene variants were once thought to explain much of the risk of most common complex diseases - the "common disease/common variant" hypothesis. GWAS, however, have made it clear that hypertension, diabetes, cardiovascular disease, depression, many cancers, and traits such as obesity are each linked to many hundreds of gene variants that individually and even collectively explain only a small fraction of the variance in disease frequency. Extensive analyses of thousands of potential gene-health outcomes often fail to match, let alone exceed, the predictive power of a few simply acquired and readily measured characteristics such as family history, neighborhood, socioeconomic circumstances, or even measurements made with nothing more than a tape measure and a bathroom scale (6). Many of the gene variants uncovered in these expensive studies are also remote from any known or plausible biological mechanism. The failure of hundreds of GWAS to find actionable relationships between exposure and disease shows that this key foundational assumption of precision medicine is unfounded.

Diagnostics. Absent the expected tight linkage between a few DNA variants and disease, the diagnostic and prognostic power of DNA testing has been limited to a few highly penetrant examples such as BRCA variants in breast cancer. The argument, therefore, has shifted to "polygenic risk scores," which use large numbers of gene variants with very small effect sizes as tools for predicting disease. But finding correlations between these risk scores and disease is only the first step in using them for population screening and early intervention. To minimize both missed cases and overdiagnosis, screening parameters such as sensitivity, specificity, and predictive value must be determined in specific populations and evidence produced that such screening improves health (7). Notably, arguments in favor of polygenic risk scores are, like traditional prediction models, probabilistic and not precise as envisioned.

The operative question, however, should not be whether genes predict but whether genes add explanatory value to what we already know. In the case of coronary heart disease, gene scores add little to traditional risk prediction models (8). Thus, a second foundational idea underpinning precision medicine has major limitations.

Pharmacogenomics. Notwithstanding some success in avoiding rare serious drug reactions, trials have largely failed to support the utility of pharmacogenomic testing for most common classes of drugs. Trials of pharmacogenomic dosing of warfarin, in which "precise" dosing is needed to balance the antithrombotic effect of the drug against the risk of severe bleeding, have shown no benefit of such testing (9). By contrast, trials of a one-size-fits-all pill containing aspirin, a statin, and an antihypertensive - the very antithesis of precision medicine - for the prevention of cardiovascular disease have shown effectiveness (10).

The polyclonal and adaptive nature of most malignancies makes demonstrating improved overall survival across a broad array of cancers challenging. A multicenter randomized trial of treatment based on tumor sequencing compared with conventional cancer treatment showed no advantage of sequencing (11), and the most recent findings of the large $(6,000$ patients screened thus far) NCI-MATCH (National Cancer Institute-Molecular Analysis for 


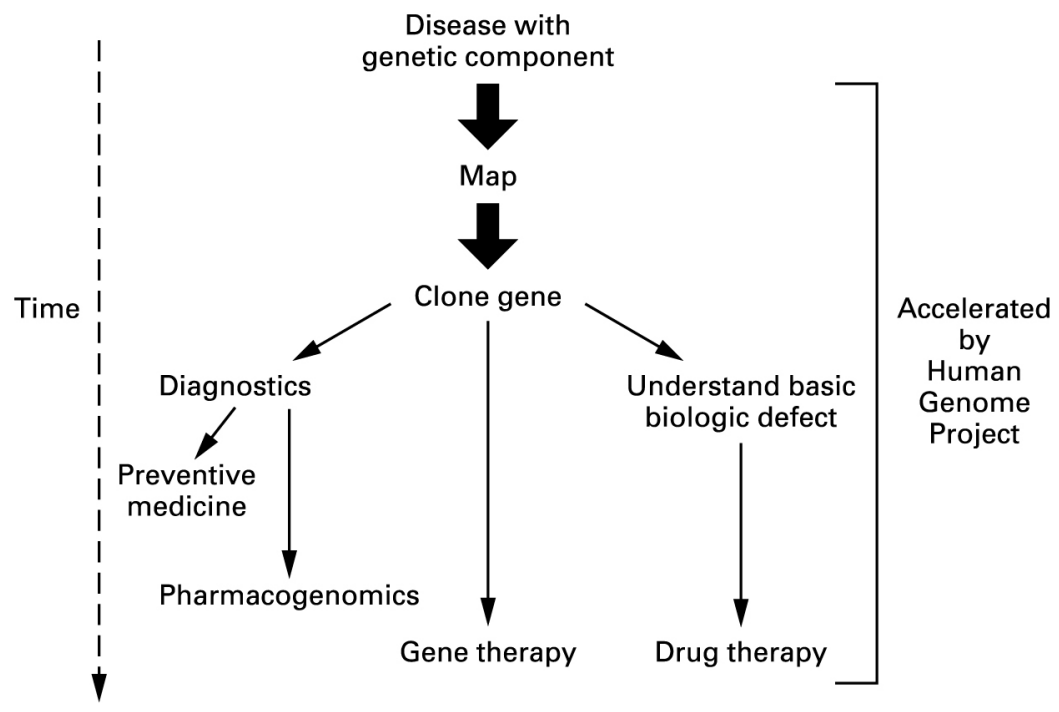

Therapy Choice) trial also fail to show benefit (12). Additionally, the polyclonal and adaptive nature of most malignancies makes demonstrating improved overall survival across a broad array of cancers challenging. Moreover, the fraction of cancer patients whose tumors demonstrate clear evidence of "targetable" signatures is very small (13).

The limits of pharmacogenomics and targeted therapy show that, while a third foundational idea underpinning precision medicine may have niche applications, the hoped-for broad successes envisioned by precision medicine advocates are unlikely.

Preventive medicine. In imagining the "gene-based medicine" of 2010, Collins, in 1999, described a young smoker who learns that his genes convey a six-fold increased risk of lung cancer. "Confronted with the reality of his own genetic data, he arrives at that crucial 'teachable moment' when a lifelong change in health-related behavior...is possible." This genetic finding "provides the key motivation for him to join a support group of persons at genetically high risk for serious complications of smoking, and he successfully kicks the habit." We must presume that smoking itself, which increases the risk of lung cancer by 20- to 100-fold, depending on cell type, has somehow failed to provide this young man a teachable moment (14).

Two assumptions are embedded in this scenario: that high-relative-risk groups will be discovered through genetic testing and that genetic data will change behavior. The first assumption, as noted above, has generally not been supported, and no gene has been discovered since 1999 that conveys a relative risk of six for lung cancer. The second assumption, that communicating genetic risk estimates will change behavior, is not supported by a meta-analysis of 18 studies (15). Thus, a fourth foundational idea, that genetic knowledge will change behavior, is also not supported by evidence, though this lack of evidence has done little to stem the tide of direct-to-consumer genetic testing.

Gene therapy. For many years the clinical application of gene therapy was stalled, though recent trials have shown promising results in several rare diseases. These successes are impressive, but their costs are enormous, and it is unclear how it will be possible to fund such therapy. The idea, expressed at the dawn of the genomic era, that gene therapy was also a possibility for the treatment of common diseases, has largely been abandoned. Thus, a fifth foundational idea central to precision medicine, that gene therapy might have implications for common diseases, has not so far been supported.

Understand the basic biologic defect and treat with drug therapy. As the expected value of many GWAS has failed to materialize, the argument for the value of human genomics has shifted from disease prediction and prevention to the use of gene variants to increase understanding of disease biology for translation into drug therapy. The hidden assumption is that the host genome is the driver of every cellular event. But the genome is as much
Figure 1. Schematic from 1999 illustrating anticipated advances in medicine that foreshadow personalized or precision medicine. We have highlighted key nodes in this figure to permit us to assess progress over the past 19 years on the six processes that were proposed to be accelerated by the Human Genome Project. Figure reproduced from The New England Journal of Medicine (license no. 4466160787447), Copyright 1999, Massachusetts Medical Society. Reprinted with permission from Massachusetts Medical Society (1) acted upon as it is actor, and the trigger for gene action is frequently an environmental stimulus, making the environment the primary cause, not the gene.

Unfortunately, the pharma pipeline has not been filled by GWAS-identified targets (16). Even where the study of genes has led to novel compounds, as with PCSK9 inhibitors, the clues have come from rare families identified on the basis of their clinical picture and not from GWAS conducted on large samples of the population (17). The new, genetically driven drugs for cystic fibrosis parallel the situation in cancer, in that they have modest effects but huge costs (18). Thus, a sixth tenet of precision medicine has yet to bear fruit, particularly for common complex diseases that occur later in life.

\section{Summary: what is success?}

The promises of precision medicine are to dramatically change patient care via individually tailored therapies and, as a result, to prevent disease, improve survival, and extend healthspan (19).

However, nearly two decades after the first predictions of dramatic success, we find no impact of the human genome project on the population's life expectancy or any other public health measure, notwithstanding the vast resources that have been directed at genomics. Exaggerated expectations of how large an impact on disease would be found for genes have been paralleled by unrealistic timelines for success, yet the promotion of precision medicine continues unabated. 
In light of the limitations of the precision medicine narrative, it is urgent that the biomedical research community reconsider its ongoing obsession with the human genome and reassess its research priorities including funding to more closely align with the health needs of our nation. We do not lack for pressing public health problems. We must counter the toll of obesity, inactivity, and diabetes; we need to address the mental health problems that lead to distress and violence; we cannot stand by while a terrible opiate epidemic ravages our country; we have to prepare conscientiously for the next influenza pandemic; we have a responsibility to prevent the ongoing contamination of our air, food, and water. Topics such as these have taken a back seat to the investment of the NIH and of many research universities in a human genome-driven research agenda that has done little to solve these problems, but has offered us promises and more promises.

\section{Acknowledgments}

The authors would like to thank their ongoing email network of some 30 outstanding scientists from many areas of biomedical research, who share papers of interest on related topics. The opinions expressed in this article are the authors' and not those of their institutions.

Address correspondence to: Michael J. Joyner, Department of Anesthesiology and Perioperative Medicine, Mayo Clinic,
Rochester, Minnesota 55905, USA. Phone: 507.255.7300; Email: joyner.michael@ mayo.edu. Or to: Nigel Paneth, Departments of Epidemiology and Biostatistics and Pediatrics and Human Development, Michigan State University, College of Human Medicine, 909 Fee Road, Room 218, East Lansing, Michigan 48824, USA. Phone: 517.884.3961; paneth@epi.msu.edu.

1. Collins FS. Shattuck lecture - medical and societal consequences of the Human Genome Project. NEngl J Med. 1999;341(1):28-37.

2. Wade N. The New York Times. A Decade Later, Genetic Map Yields Few New Cures. The New York Times. June 12, 2010. https://www.nytimes. com/2010/06/13/health/research/13genome. html. Accessed December 20, 2018.

3. Snyderman R, Meade C, Drake C. Value of personalized medicine. JAMA. 2016;315(6):613.

4. Letai A. Functional precision cancer medicine-moving beyond pur genomics. Nat Med. 2017;23(9):1028-1035.

5. SoRelle JA, Thodeson DM, Arnold S, Gotway G, Park JY. Clinical utility of reinterpreting previously reported genomic epilepsy test results for pediatric patients [published online ahead of print November 5, 2018]. JAMA Pediatr. https:// doi.org/10.1001/jamapediatrics.2018.2302.

6. Loos RJF, Janssens A. Predicting polygenic obesity using genetic information. Cell Metab. 2017;25(3):535-543.

7. Saracci R. Epidemiology in wonderland: Big Data and precision medicine. Eur J Epidemiol. 2018;33(3):245-257.

8. Morris RW, et al. Marginal role for 53 common genetic variants in cardiovascular disease prediction. Heart. 2016;102(20):1640-1647.

9. Stergiopoulos K, Brown DL. Genotype-guided vs clinical dosing of warfarin and its analogues: meta-analysis of randomized clinical trials. JAMA Intern Med. 2014;174(8):1330-1338.
10. Selak V, et al. Reaching cardiovascular prevention guideline targets with a polypill-based approach: a meta-analysis of randomised clinical trials. Heart. 2019;105(1):42-48.

11. Le Tourneau C, et al. Molecularly targeted therapy based on tumour molecular profiling versus conventional therapy for advanced cancer (SHIVA): a multicentre, open-label, proof-ofconcept, randomised, controlled phase 2 trial. Lancet Oncol. 2015;16(13):1324-1334.

12. Eckhardt SG, Lieu C. Is precision medicine an oxymoron? [published online ahead of print November 8, 2018]. JAMA Oncol. https://doi. org/10.1001/jamaoncol.2018.5099.

13. Marquart J, Chen EY, Prasad V. Estimation of the percentage of US patients with cancer who benefit from genome-driven oncology. JAMA Oncol. 2018;4(8):1093-1098.

14. Pesch B, et al. Cigarette smoking and lung cancer - relative risk estimates for the major histological types from a pooled analysis of case-control studies. Int J Cancer. 2012;131(5):1210-1219.

15. Hollands GJ, et al. The impact of communicating genetic risks of disease on risk-reducing health behaviour: systematic review with meta-analysis. BMJ. 2016;352:11102.

16. Scannell JW, Bosley J. When quality beats quantity: decision theory, drug discovery, and the reproducibility crisis. PLoS One. 2016;11(2):e0147215.

17. Farnier M. PCSK9: From discovery to therapeutic applications. Arch Cardiovasc Dis. 2014;107(1):58-66.

18. Sharma D, Xing S, Hung YT, Caskey RN, Dowell ML, Touchette DR. Cost-effectiveness analysis of lumacaftor and ivacaftor combination for the treatment of patients with cystic fibrosis in the United States. Orphanet J Rare Dis. 2018;13(1):172.

19. Francis S.Collins (@NIHDirector), “I am here at the \#JoinAllofUs Twitter chat! Ask me questions on \#PrecisionMedicine \& the @AllofUsResearch Program for the next 30 minutes," Twitter, May 8, 2018, 10:31 am, https://twitter.com/ nihdirector/status/993906139036356608. Accessed December 20, 2018. 